\title{
Experimental Investigation of Thermal Conductivity and Effusivity of Ferrite Based Nanofluids under Magnetic Field
}

\author{
Ashok K. Singh ${ }^{1}$ and Vijay S. Raykar ${ }^{2}$ \\ ${ }^{1}$ DRDO Center for Piezoceramics and Devices, ARDE, Pashan, Pune 411021, India \\ ${ }^{2}$ Nanomaterials and Sensors Laboratory, Defence Institute of Advanced Technology (DU), Girinagar, Maharashtra, Pune 411025, India \\ Correspondence should be addressed to Ashok K. Singh; aksingh@diat.ac.in
}

Received 23 May 2013; Accepted 12 June 2013

Academic Editors: D. R. Chen, S. Maksimenko, and D. K. Yi

Copyright (c) 2013 A. K. Singh and V. S. Raykar. This is an open access article distributed under the Creative Commons Attribution License, which permits unrestricted use, distribution, and reproduction in any medium, provided the original work is properly cited.

We investigate the effect of magnetic field $(H)$ on the thermal conductivity $(\lambda)$ and effusivity $(\varepsilon)$ of cobalt ferrite based nanofluids having different concentrations $(\phi)$. Cobalt ferrite nanoparticles (NPs) have been synthesized using the microwave assisted method. At high volume fraction of cobalt ferrite nanoparticles in water $(\phi>0)$, both thermal parameters have been found to be suppressed relative to $\phi$ and $\lambda$ of water in the absence of $H$. However, it is seen that percentage values of the effusivity in perpendicular field direction show negative to positive variation, and thermal conductivity in parallel field direction shows negative to zero variation.

\section{Introduction}

Enhancement in $\lambda$ of magnetic nanofluid under the effect of applied $H$ has been reported by Philip et al. [1]. It is also reported that these properties are suppressed for doped metal oxide based nanofluids and with the application of $H[2,3]$. Both these behaviors in thermal transport properties are the outcome of different nature of phonon transport in metal, metal oxide, and doped metal oxide nanoparticle (NP) based nanofluids (NFs). Experimental data about $\lambda$ enhancement alone does not reflect that other transport properties $(\varepsilon$, specific heat) are also affected in a similar manner with respect to $H$. It was pointed out that this ambiguity creates the problem when looking for efficient nanofluids in terms of transport properties [4]. If we can characterize nanofluids with respect to the previous three transport properties, with and without application of $H$, then we can better select the most appropriate nanofluid for desired application.

Effusivity, also named thermal admittance or contact coefficient, is defined as the material's ability to exchange heat with surroundings. It is defined through $[5,6]$

$$
\varepsilon=\sqrt{\lambda \rho c}=\frac{\lambda}{\sqrt{\alpha}}
$$

where $\lambda$ is the thermal conductivity, $\rho$ is the density, $c$ is the specific heat, $\alpha$ is the thermal diffusivity, and $\varepsilon$ has the dimensions of $W s^{1 / 2} \mathrm{~cm}^{-2} C^{-1}$. Potential applications of NFs depend upon the enhancement in transport properties and in turn on their behavior under the applied $H$. Hence, it is of great importance to corroborate the reported experimental results $[2,3]$, which is the aim of the present study.

Dadarlat et al. [2] have used high accuracy photopyroelectric technique to obtain thermal properties of cobalt ferrite $\left(\mathrm{CoFe}_{2} \mathrm{O}_{4}\right)$ based NFs, and compared with the other studied magnetic fluids, they also reported that transport properties of $\mathrm{CoFe}_{2} \mathrm{O}_{4}$ NFs have been degraded with increasing volume fraction $(\phi)$. Djurek et al. [3] also reported decrease in $\lambda$ of $\mathrm{CoFe}_{2} \mathrm{O}_{4}$ NFs with application of $H$. However, none of the authors gives an explanation of the mechanism for these suppressed thermal properties and possibilities to enhance it with the application of $H$. For example, Fe NPs in base fluid were found to exhibit enhanced $\lambda$ at applied $H$, and studies with varying directions of applied $H$ indicated that this could be associated with a chain like structure formation involving field gradient parallel to thermal gradient, analogous to the anisotropic transport properties of magnetorheological fluids [7-9]. Similarly, Lajvardi et al. [10] have reported that enhancement in convective heat transfer performance of 
$\mathrm{Fe}_{3} \mathrm{O}_{4}$ based ferrofluid under the application of magnetic field.

In this paper microwave assisted method for synthesis of cobalt ferrite nanoparticles (NPs) has been used, and synthesized NPs have been used for preparation of water based nanofluids. Here, we report how we can transform thermal property negative variation (in \% relative to base fluid) in $\varepsilon^{\| \prime}$ (parallel field direction) of $\mathrm{CoFe}_{2} \mathrm{O}_{4}$ based fluids to positive one with the application of perpendicular $H$. The $\varepsilon^{\perp}$ (perpendicular field direction) measurements have revealed a high enhancement ratio for given $\phi$. Our results show that the $\lambda^{\|}$has the same order of magnitude as that of water at low $\phi$ and which decreases with increase in the $\phi$.

\section{Experimental Details}

2.1. Materials. The starting materials used in this work were cobalt nitrate hexahydrate $\left(\mathrm{Co}\left(\mathrm{NO}_{3}\right)_{2} \cdot 6 \mathrm{H}_{2} \mathrm{O}\right)$, ferric nitrate hexahydrate $\left(\mathrm{Fe}\left(\mathrm{NO}_{3}\right)_{2} \cdot 6 \mathrm{H}_{2} \mathrm{O}\right)$, sodium hydroxide $(\mathrm{NaOH})$, trisodium citrate, and nitric acid. All chemicals were used as received without further purification.

2.2. Synthesis Procedure. The $\mathrm{CoFe}_{2} \mathrm{O}_{4}$ NFs were prepared by using a previously described procedure [11] with slight modifications. In a typical process, sodium hydroxide $(\mathrm{NaOH}, 16 \mathrm{gm} / 40 \mathrm{~mL})$ was added to a mixture of cobalt $\left(\mathrm{Co}\left(\mathrm{NO}_{3}\right)_{2} \cdot 6 \mathrm{H}_{2} \mathrm{O}, 4 \mathrm{gm} / 100 \mathrm{~mL}\right)$ and ferric nitrate $\left(\mathrm{Fe}\left(\mathrm{NO}_{3}\right)_{2} \cdot 6 \mathrm{H}_{2} \mathrm{O}, 12 \mathrm{gm} / 100 \mathrm{~mL}\right)$ under vigorous stirring. A dispersion of a nonmagnetic amorphous precipitate was obtained. Then, the solution was poured into the reaction flask. The resulting solution was heated for $40 \mathrm{~min}$. at $80^{\circ} \mathrm{C}$ in microwave oven at medium power and then cooled to a room temperature. The magnetic precipitate was collected via centrifugation and washed with deionised water for several times. The magnetic precipitate was then dispersed in a solution of nitric acid $(200 \mathrm{~mL} ; 1 \mathrm{~mL} / \mathrm{L})$ for $5 \mathrm{~min}$. The precipitate was again separated and was stirred in a trisodium citrate solution $(7 \mathrm{gm} / 200 \mathrm{~mL})$ at $80^{\circ} \mathrm{C}$, for $30 \mathrm{~min}$. in a microwave oven at medium power. This sample is NF with $\phi=1$ (100 mL, original concentration). The NFs with $\phi=0.1$, 0.01 were subsequently prepared by diluting the NF $(\phi=1$, $100 \mathrm{~mL}$ ) by adding $90 \mathrm{~mL}$ of water to $10 \mathrm{~mL}(\phi=1)$ and 99 $\mathrm{mL}$ of DI water to $1 \mathrm{~mL}(\phi=1)$, respectively.

2.3. Characterization. The size and morphology of the $\mathrm{CoFe}_{2} \mathrm{O}_{4}$ NPs were determined using scanning electron microscopy (SEM) (JEOL ASM 6360A). The average particle sizes of the $\mathrm{CoFe}_{2} \mathrm{O}_{4}$ NPs were measured by particle size analyzer (Nicomp ZLS 380).

2.4. Measurement of Thermal Effusivity and Conductivity. Thermal effusivity is measured using plane heat source technique as described in [12]. Alignment (perpendicular, parallel) of sensor in the presence of external $H$ is shown in Figure 1. Thermal conductivity was measured using hot wire transient method with platinum wire (diameter $=30 \times$ $10^{-6} \mathrm{~m}$ ) and data acquisition system DataTaker (DT 80) as a function of parallel applied $H$ [10]. In our experimental setup

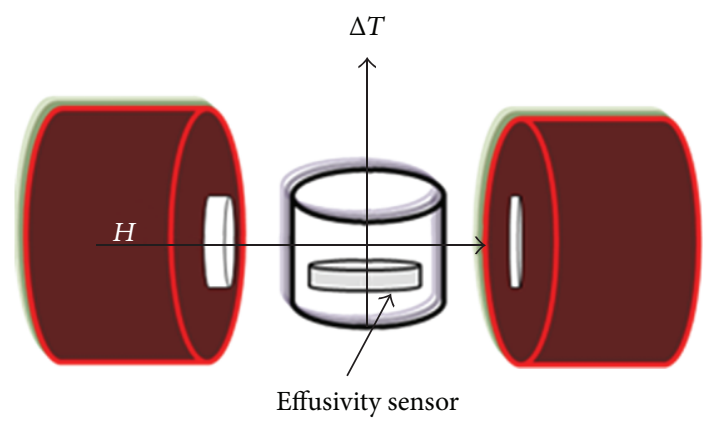

(a)

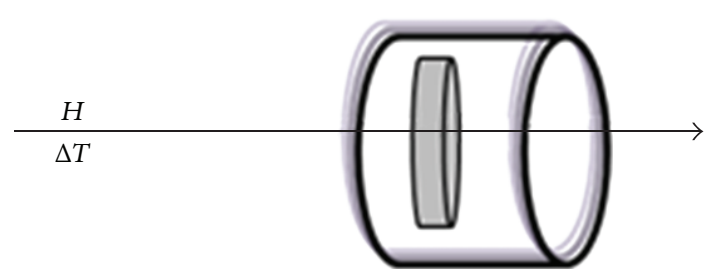

(b)

FIgURE 1: The experimental arrangement for $\varepsilon$ measurement of $\mathrm{CoFe}_{2} \mathrm{O}_{4}$ NFs in (a) perpendicular and (b) parallel $H$ directions.

$\lambda$ measurement in perpendicular direction is not possible due to restriction of sensor size and experimental setup. Measurement system has been calibrated by measuring $\varepsilon$ and $\lambda$ of known liquids, that is, water and ethylene glycol.

\section{Results and Discussion}

The SEM image (Figure 2(a)) of the drop casted $\mathrm{CoFe}_{2} \mathrm{O}_{4} \mathrm{NF}$ on glass substrate shows randomly distributed agglomerates of NPs on the glass surface. The diameter is in the range of 70$80 \mathrm{~nm}$, which was corroborated using particle size analysis (Figure 2(b)).

The obtained values of $\lambda^{\|}$exhibit several interesting features (Figure 3). NFs with $\phi=1$ showed a marginal rise where the effect of $H$ was manifested, whereas NFs with $\phi \leq 0.5$ showed constant values of $\lambda^{\|}$without any appreciable effect of $H$. We note that these values $(-80 \%$ for $\phi=1)$ of $\lambda$ which are below $\lambda$ of water were not observed in some of the previous studies of magnetic NFs, possibly due to the low $\lambda$ of doped metal oxides. The applied $H$ range varied from 0 to $0.12 \mathrm{~T}$ in steps of $0.04 \mathrm{~T}$, and change in $\lambda^{\|}$is about $-8 \%$ in case of $\phi=1$. It is observed that $\lambda^{\|}$decreases appreciably with increase in $\phi \geq 1$ and rises to about $\lambda$ of water for low values of $\phi$. At $\phi=0.01, \lambda^{\|}$shows $-1 \%$ or less change under the effect of $H$. As $\phi$ is lowered $(<0.01)$, all the nanofluids begin to show water like behavior.

We now look at the $\varepsilon^{\perp}$ variation of the NFs with applied $H$ (Figure 4). For $\phi=1$ and $0.1, \varepsilon^{\perp}$ exhibited a much more pronounced effect of $H$ than that observed in the $\lambda$ studies (compare Figures 3 and 4). For $\phi=1$, variation in $\varepsilon^{\perp}$ is about $47 \%$ in contrast to $8 \%(\phi=1)$ as measured for $\lambda$ with applied $H$. However, the variation for $\phi=0.01$ is approximately the same for both $\varepsilon^{\perp}$ and $\lambda^{\|}$with applied $H$. The most interesting 


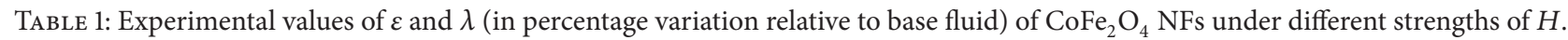

\begin{tabular}{lcccc}
\hline$H$ & $\varepsilon^{\|}$(in parallel $H$ direction) & $\varepsilon^{\perp}$ (in perpendicular $H$ direction) & $\lambda^{\|}$(in parallel $H$ direction) & $\lambda^{\perp}$ (in perpendicular $H$ direction) \\
\hline 0.00 & -28.42 & -27.45 & -79.32 & \\
0.04 & -24.84 & +05.51 & -75.86 & Not measured (experimental \\
0.08 & -19.93 & +11.87 & -73.63 & limitation) \\
0.12 & -13.69 & +19.63 & -72.67 & \\
\hline
\end{tabular}

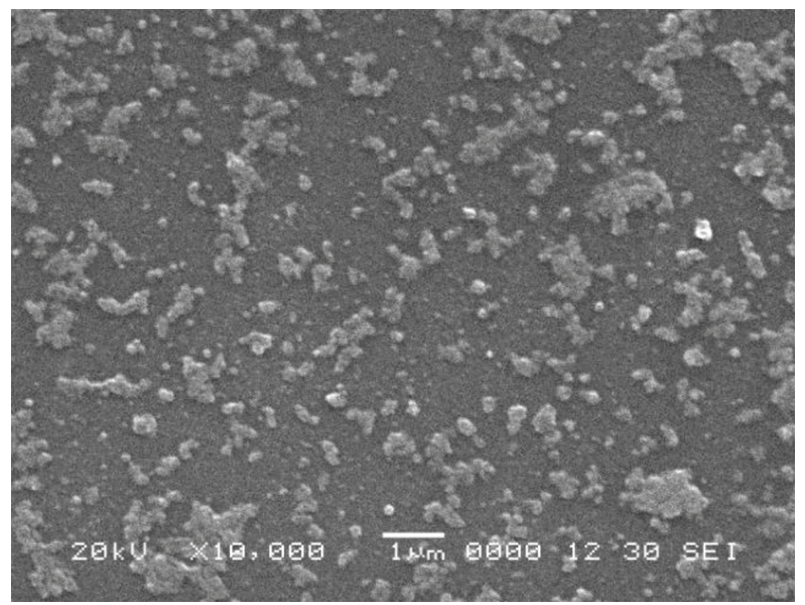

(a)

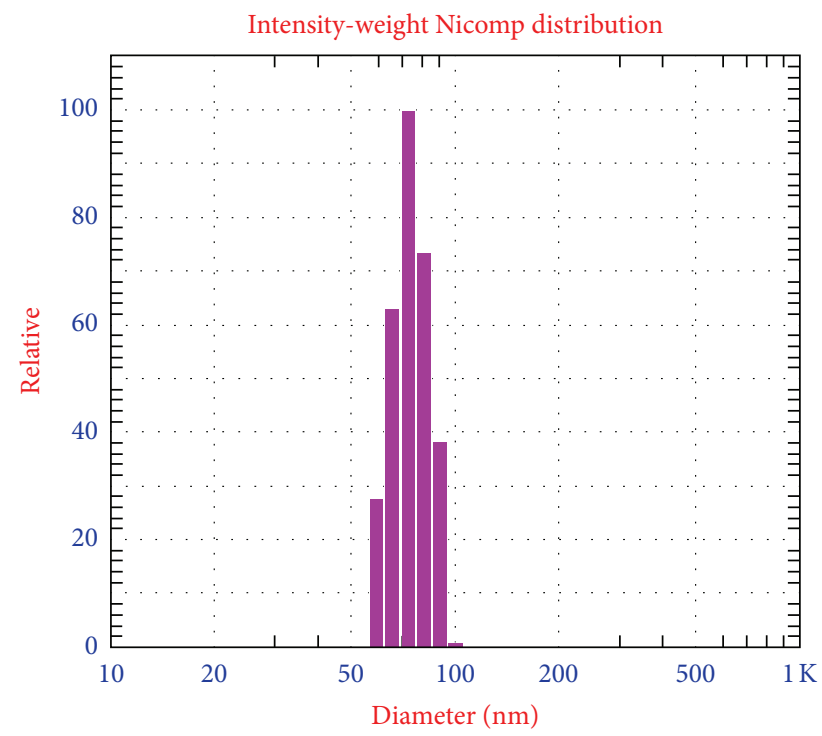

(b)

FIGURE 2: SEM image of $\mathrm{CoFe}_{2} \mathrm{O}_{4} \mathrm{NPs}(\mathrm{a})$; particles size distribution of nanoparticles (NPs) based on number-weight distribution (b).

fact is the transition to completely positive values of $\varepsilon^{\perp}$ for $\phi \geq$ 0.1 , even though $H$ is perpendicular to thermal gradient. On the other hand, with application of parallel field the variation in $\varepsilon$ was found to be varying from $-28 \%$ to $-14 \%$ in the case of $\phi=1$ ( $\varepsilon^{\|}$, see Figure 5). For $\phi=1$ and 0.1 no appreciable variation was found in $\varepsilon^{\|}$values $(-14$ to $-10 \%,-11$ to $-8 \%)$. The results for $\phi=1$ are shown in Table 1 .

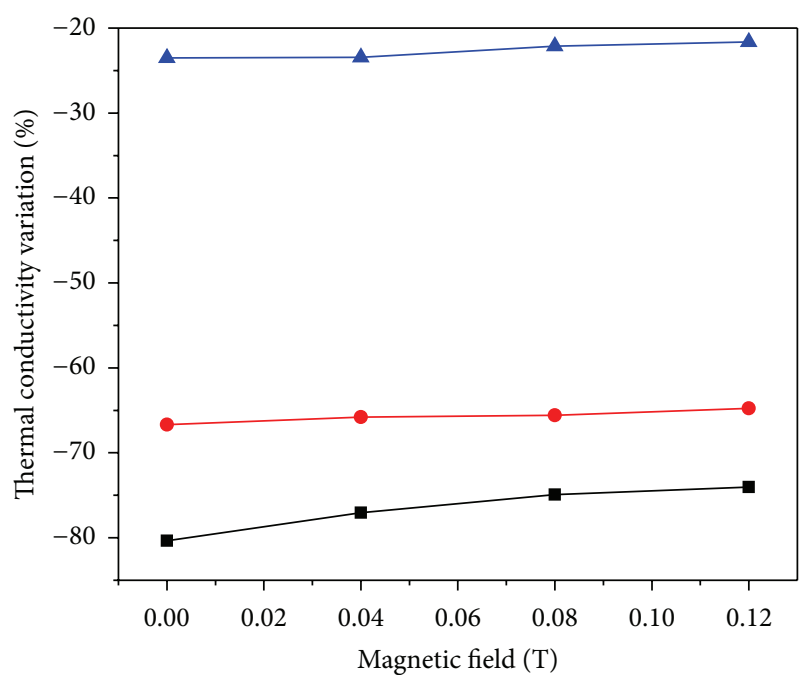

$$
\begin{aligned}
& \longrightarrow \phi=1 \\
& \longrightarrow \phi=0.1 \\
& \longrightarrow \phi=0.01
\end{aligned}
$$

FIgURE 3: The $\lambda^{\|}$variation as a function of $H$ for $\mathrm{CoFe}_{2} \mathrm{O}_{4} \mathrm{NFs}$ for $\phi=1, \phi=0.1$, and $\phi=0.01$.

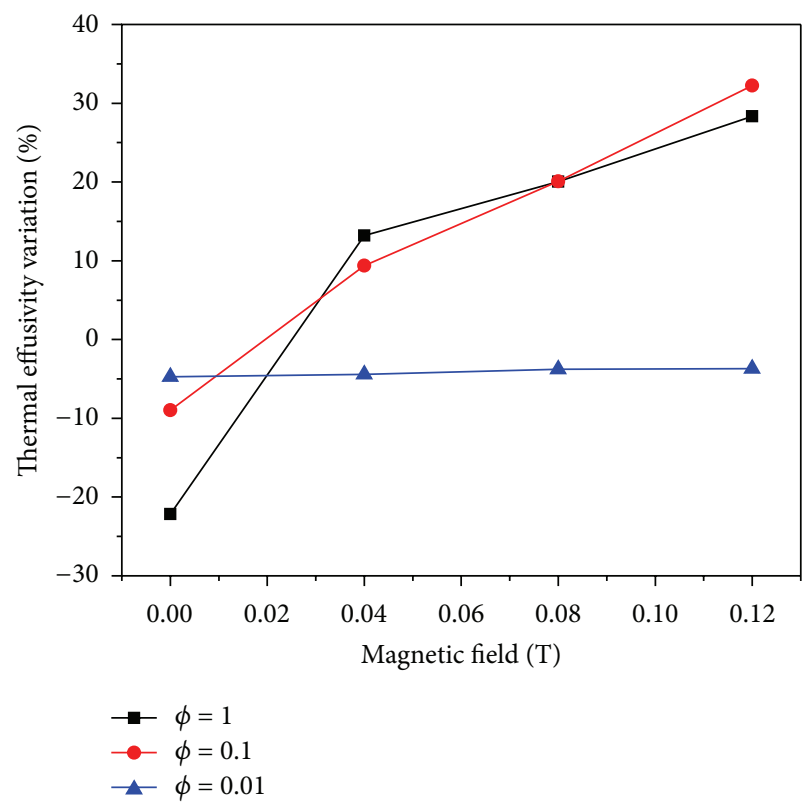

FIgURE 4: The $\varepsilon^{\perp}$ variation as a function of $H$ for $\mathrm{CoFe}_{2} \mathrm{O}_{4} \mathrm{NFs}$ for $\phi=1, \phi=0.1$, and $\phi=0.01$. 


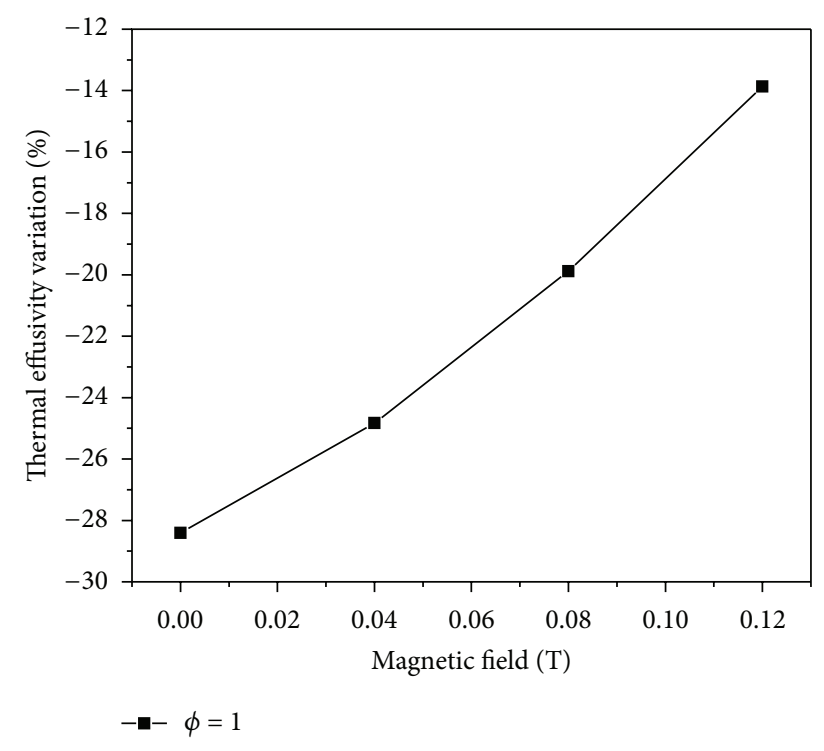

FIgURE 5: The $\varepsilon^{\|}$variation as a function of $H$ for $\mathrm{CoFe}_{2} \mathrm{O}_{4} \mathrm{NFs}(\phi=$ $1)$.

This indicates that $\varepsilon$ is sensitive to the direction of applied $H$ to thermal gradient. It is also interesting to look for analogy between the $\varepsilon^{\perp}$ increment and $\lambda^{\|}$increment as reported by Li et al. $[7,8]$. They considered, in particular, $\lambda^{\perp}$ and $\lambda^{\|}$of Fe water system and showed that $\lambda^{\|}>\lambda^{\perp}$ due to chain like structure formation when applied field $(H)$ is parallel. Unfortunately, a direct comparison of $\lambda^{\|}, \lambda^{\perp}$ with $\varepsilon^{\|}, \varepsilon^{\perp}$ is not possible due to the use of different kind of sensors and heat transfer phenomena. Qualitatively, this change in $\varepsilon^{\perp}$ may be associated with effect of $\mathrm{H}$ on $\mathrm{CoFe}_{2} \mathrm{O}_{4} \mathrm{NFs}$, which reflects an increase in viscosity due to coherent interaction between NPs with structure formation [7].

The phonon contribution affects the overall transport properties of material. Its influence on $\lambda$ has been studied in detail and was attributed to generation of cation vacancies (lattice vacancies) in host through doping of other materials which resulted in increase in phonon scattering and decrease in mean free path [13]. In the case of polycrystalline material (cobalt ferrite), the presence of interfacial regions known as grain boundaries affects the average $\lambda$ by scattering of phonons [14]. Both electrons and phonons are effectively scattered in nanoprecipitates of different chemical compositions [15]. This justifies the observed negative variation in $\varepsilon$ and $\lambda$ of $\mathrm{CoFe}_{2} \mathrm{O}_{4}$ NFs with added volume fraction.

Experimental results of dependence of $\varepsilon$ of magnetic NFs with variation in $H$ were not available, and as mentioned previously, our experiments showed $\varepsilon^{\perp}>\varepsilon^{\|}$. We believe this variability was due to the regular lattice formation within cobalt ferrite NPs with decrement in lattice period (i.e., small unit cell parameters) with perpendicular $H$ field, causing less phonon scattering with increase in $H$. This lattice structure provides longer mean free path lengths for heat carrying phonons [16]. $H$ independence of $\lambda^{\|}$and $\varepsilon^{\|}$can be attributed to no structure formation in parallel field direction due to anisotropic behavior of $\mathrm{CoFe}_{2} \mathrm{O}_{4} \mathrm{NFs}$.

\section{Conclusions}

Both $\lambda$ and $\varepsilon$ of $\mathrm{CoFe}_{2} \mathrm{O}_{4}$ NFs were found to decrease (below those of water) with increasing concentration $(\varphi)$. This decrement can be attributed to poor conducting (thermally) nature of doped (Co) metal oxide $\left(\mathrm{Fe}_{2} \mathrm{O}_{4}\right)$ NPs. $\varepsilon^{\perp}$ shows large positive variation for $\varphi=1$ and 0.1 under the effect of $H$ as compared to $\varepsilon^{\|}$. Enhancement in $\varepsilon$ in perpendicular field direction can be attributed to the increase in rate of phonon transfer. It is expected that in future by incorporating structures with dopants in NFs, we will be able to tune the interfacial scattering mechanism of phonons with external fields to enhance NFs transport properties.

\section{Acknowledgments}

The authors are thankful to Director of ARDE, Pune, India and Vice-Chancellor, DIAT, Girinagar, Pune, India for granting permission to publish this work. Authors are also thankful to University of Pune for providing the SEM and XRD of samples.

\section{References}

[1] J. Philip, P. D. Shima, and B. Raj, "Nanofluid with tunable thermal properties," Applied Physics Letters, vol. 92, no. 4, Article ID 043108, 3 pages, 2008.

[2] D. Dadarlat, C. Neamtu, M. Streza et al., "High accuracy photopyroelectric investigation of dynamic thermal parameters of $\mathrm{Fe}_{3} \mathrm{O}_{4}$ and $\mathrm{CoFe}_{2} \mathrm{O}_{4}$ magnetic nanofluids," Journal of Nanoparticle Research, vol. 10, no. 8, pp. 1329-1336, 2008.

[3] I. Djurek, A. Žnidaršič, A. Košak, and D. Djurek, "Thermal conductivity measurements of the $\mathrm{CoFe}_{2} \mathrm{O}_{4}$ and $\gamma-\mathrm{Fe}_{2} \mathrm{O}_{3}$ based nanoparticle ferrofluids," Croatica Chemica Acta, vol. 80, no. 34, pp. 529-532, 2007.

[4] V. S. Raykar and A. K. Singh, "Dispersibility dependence of thermal conductivity of carbon nanotube based nanofluids," Physics Letters A, vol. 374, no. 45, pp. 4618-4621, 2010.

[5] E. Marín, "The role of thermal properties in periodic timevarying phenomena," European Journal of Physics, vol. 28, no. 3, pp. 429-445, 2007.

[6] S. D. Park, S. W. Lee, S. Kang et al., "Effects of nanofluids containing graphene/graphene-oxide nanosheets on critical heat flux," Applied Physics Letters, vol. 97, no. 2, Article ID 023103, 2010.

[7] Q. Li, Y. Xuan, and J. Wang, "Experimental investigations on transport properties of magnetic fluids," Experimental Thermal and Fluid Science, vol. 30, no. 2, pp. 109-116, 2005.

[8] B. N. Reinecke, J. W. Shan, K. K. Suabedissen, and A. S. Cherkasova, "On the anisotropic thermal conductivity of magnetorheological suspensions," Journal of Applied Physics, vol. 104, no. 2, Article ID 023507, 7 pages, 2008.

[9] S. P. Patel, J. C. Pivin, A. K. Chawla, R. Chandra, D. Kanjilal, and L. Kumar, "Room temperature ferromagnetism in $\mathrm{Zn}_{1-x} \mathrm{Co}_{x} \mathrm{~S}$ thin films with wurtzite structure," Journal of Magnetism and Magnetic Materials, vol. 323, no. 22, pp. 2734-2740, 2011.

[10] M. Lajvardi, J. Moghimi-Rad, I. Hadi et al., "Experimental investigation for enhanced ferrofluid heat transfer under magnetic field effect," Journal of Magnetism and Magnetic Materials, vol. 322, no. 21, pp. 3508-3513, 2010. 
[11] F. Royer, D. Jamon, J. J. Rousseau et al., "Magneto-optical properties of $\mathrm{CoFe}_{2} \mathrm{O}_{4}$ ferrofluids. Influence of the nanoparticle size distribution," Progress in Colloid and Polymer Science, vol. 126, pp. 155-158, 2004.

[12] V. S. Raykar and A. K. Singh, "Thermal and rheological behavior of acetylacetone stabilized $\mathrm{ZnO}$ nanofluids," Thermochimica Acta, vol. 502, no. 1-2, pp. 60-65, 2010.

[13] M. I. Abd El-Ati, "Thermal conductivity of $\mathrm{Zn}$ doped $\mathrm{CoFe}_{2} \mathrm{O}_{4}$ ferrites," Phase Transitions, vol. 46, no. 4, pp. 209-215, 1994.

[14] C. Poulier, D. S. Smith, M. Viana, and J. Absi, "Evolution of thermophysical characteristics in tin oxide: from pressed powder compact to fired porous body," Journal of the American Ceramic Society, vol. 91, no. 3, pp. 965-969, 2008.

[15] Y. Lan, A. J. Minnich, G. Chen, and Z. Ren, "Enhancement of thermoelectric figure-of-merit by a bulk nanostructuring approach," Advanced Functional Materials, vol. 20, pp. 357-376, 2010.

[16] J. R. Sootsman, D. Y. Chung, and M. G. Kanatzidis, "New and old concepts in thermoelectric materials," Angewandte Chemie-International Edition, vol. 48, no. 46, pp. 8616-8639, 2009. 

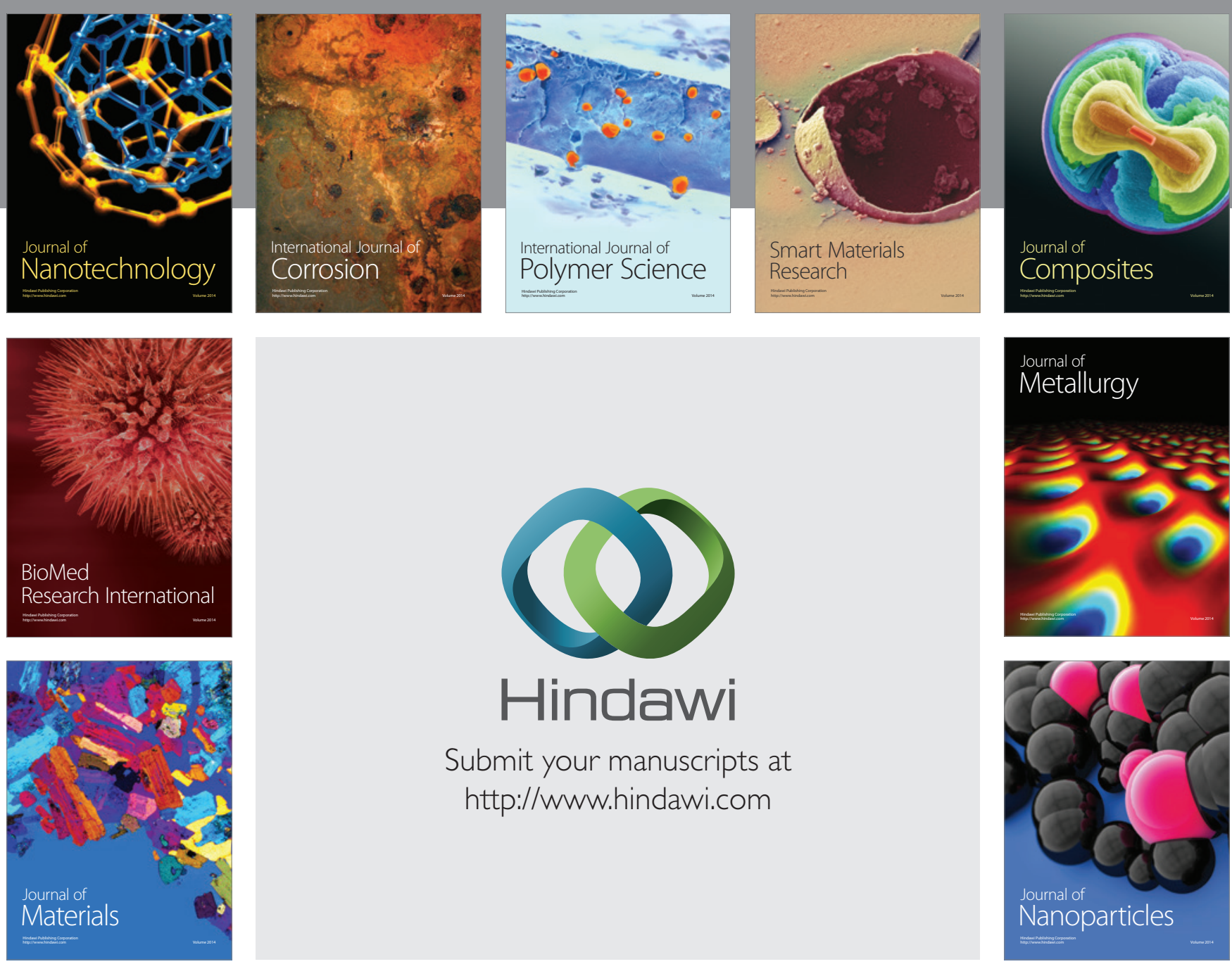

Submit your manuscripts at http://www.hindawi.com
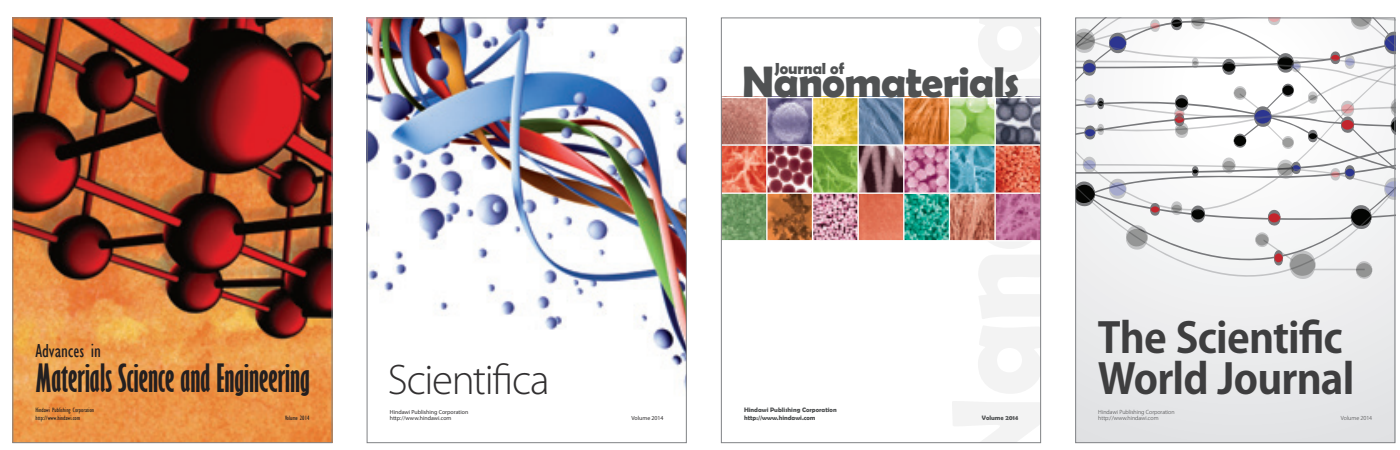

\section{The Scientific World Journal}
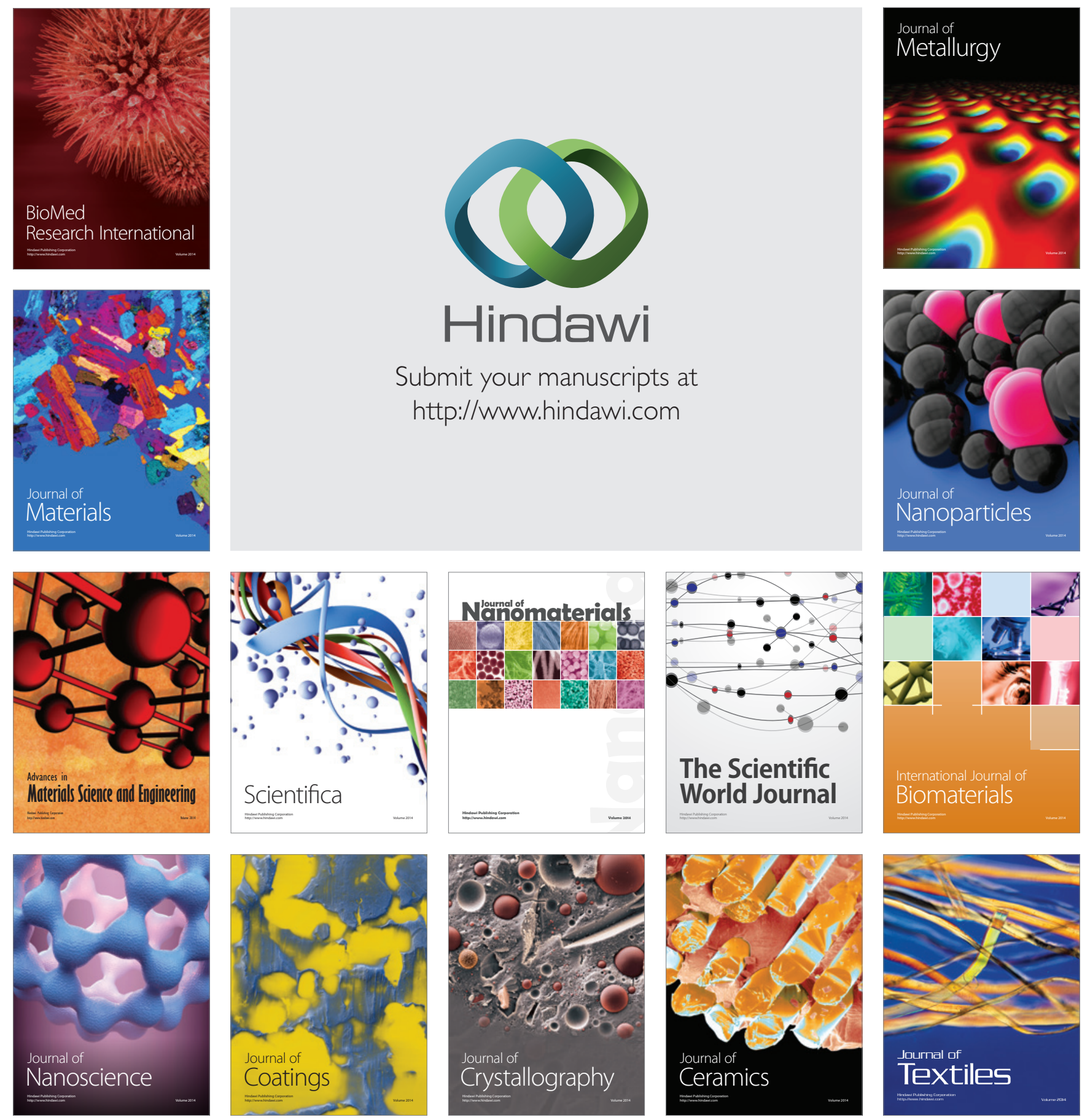